\title{
Molecular epidemiology and drug resistance prevalence of strains from newly diagnosed HIV-1 patients in Northern Greece during 2009-2010
}

\author{
Zoe Antoniadou, loanna Kousiappa*, Johana Hezka, Lemonia Skoura, Simeon Metallidis, Pavlos Nikolaidis, \\ Nicolaos Malisiovas, Leondios G Kostrikis
}

From 17th International Symposium on HIV and Emerging Infectious Diseases (ISHEID) Marseille, France. 23-25 May 2012

\section{Introduction}

As part of a continuing effort to monitor the molecular epidemiology of HIV-1 in northern Greece, in this study we determined the genetic diversity and the prevalence of drug resistance transmission among HIV-1 strains isolated from 94 newly-diagnosed untreated consenting patients in the period 2009 to 2010.

\section{Materials and methods}

Peripheral blood mononuclear cells (PBMC's) and plasma were collected from the patients at the AIDS National Reference Laboratory of Northern Greece. Plasma RNA encoding partial pol(protease and reverse transcriptase) was amplified using TRUGENE HIV-1 genotyping assay (Siemens). Antiretroviral drug resistance prevalence was estimated by using the HIVseq program (HIV Drug Resistance Database, Stanford University). PBMC's DNA encoding partial env(gp120) V3-loop region was amplified by nested PCR and sequenced using an in-house tropism assay at the Department of Biological Sciences, University of Cyprus. Phylogenetic analysis was performed and trees were constructed for each region by using neighbor-joining with Kimura two-parameter method by means of MEGA v5.0 software.

\section{Results}

The media age of patients is 32 years with the percentage of male infection at $89.4 \%$. The main risk groups were homosexual contact (73.4\%) and heterosexual contact (17.0\%). The phylogenetic analysis indicated B and A1 as the dominant subtypes, $47.8 \%$ and $41,5 \%$ respectively, followed by subtype C, CRF02_AG (3,2\% each), CRF04_cpx (2,1\%) and subtypes F1 and G (1,1\% each). Twenty-one clusters showed epidemiologically linked HIV-1 patients. Resistance mutations to protease inhibitors were found in three individuals, while high resistance associated mutations to reverse transcriptase inhibitors (NRTI's/NNRTI's) were shown in sixteen patients. Tropism testing indicated most of the HIV-1 strains as R5-tropic $(96 \%)$.

\section{Conclusions}

These newly found data demonstrate a heterogeneous epidemiological status of HIV-1 in northern Greece during 2009-2010, with subtype B and A1 being the dominant subtypes in relation to the other subtypes. The prevalence of antiretroviral resistance mutations is high among the newly diagnosed untreated patients $(17 \%)$ in comparison with other European countries (10\%).

Published: 25 May 2012

doi:10.1186/1742-4690-9-S1-P30

Cite this article as: Antoniadou et al:: Molecular epidemiology and drug resistance prevalence of strains from newly diagnosed HIV-1 patients in Northern Greece during 2009-2010. Retrovirology 2012 9(Suppl 1):P30.

* Correspondence: kousiappa.ioanna@ucy.ac.cy

University of Cyprus, Nicosia, Cyprus 